\title{
BMJ Open Cost-effectiveness and value-based prices of the 9-valent human papillomavirus vaccine for the prevention of cervical cancer in China: an economic modelling analysis
}

$\mathrm{Y}$ Jiang, ${ }^{1}$ Weiyi $\mathrm{Ni}^{2}$ Jing $\mathrm{Wu}$ (D) ${ }^{3}$

To cite: Jiang Y, Ni W, Wu J. Cost-effectiveness and valuebased prices of the 9-valent human papillomavirus vaccine for the prevention of cervical cancer in China: an economic modelling analysis. BMJ Open 2019;9:e031186. doi:10.1136/ bmjopen-2019-031186

- Prepublication history and additional material for this paper are available online. To view these files, please visit the journal online (http://dx.doi. org/10.1136/bmjopen-2019031186).

Received 20 April 2019 Revised 12 October 2019 Accepted 05 November 2019

Check for updates

(C) Author(s) (or their employer(s)) 2019. Re-use permitted under CC BY-NC. No commercial re-use. See rights and permissions. Published by BMJ.

${ }^{1}$ School of Public Health (Shenzhen), Sun Yat-Sen University, Shenzhen,

Guangdong, China

${ }^{2}$ Department of Pharmaceutical and Health Economics, University of Southern California, Los Angeles, California, USA

${ }^{3}$ School of Pharmaceutical Science and Technology, Tianjin University, Tianjin, China

Correspondence to Dr Jing Wu; jingwu@tju.edu.cn

\section{ABSTRACT}

Objectives To evaluate the cost-effectiveness of the 9-valent human papillomavirus (HPV) vaccine for the prevention of cervical cancer in China.

Design Health economic modelling using the Papillomavirus Rapid Interface for Modelling and Economics (PRIME) model populated with China-specific data.

Setting Individual cervical cancer prevention in China using the 9-valent HPV vaccine from the perspective of private sector purchasers in relation to receiving other HPV vaccines and not receiving vaccination for 16 -year-old girls in China who had not been previously infected with HPV.

Participants Not applicable.

Interventions Vaccination using the 9-valent, the quadrivalent and the bivalent vaccines.

Primary outcome measure Incremental costs per disability-adjusted life year (DALY) prevented.

Results In the base case, the incremental costs per DALY prevented were, respectively, US\$35000 and US\$50 455 compared with the quadrivalent and the bivalent vaccines, both of which were above the cost-effective threshold of US\$25 920/DALY prevented. To be cost-effective in these comparisons, the 9 -valent vaccine should be priced at $\$ 550$ and $\$ 450$ for the full doses, respectively. To be highly cost-effective, the price thresholds were $\$ 435$ and $\$ 335$. The incremental costs per DALY prevented in relation to no vaccination was US\$23 012, making the 9-valent vaccine marginally cost-effective. The results were robust in most one-way sensitivity analyses including changing vaccination age to 13 and 26 years.

Conclusions At the current price, the 9-valent HPV vaccine is not cost-effective compared with the quadrivalent and the bivalent vaccines for young girls in China who had not been previously infected with HPV. Policymakers and clinicians should keep potential vaccine recipients informed about the economic profile of the 9-valent vaccine and carefully consider expanding its use in China at the current price.

\section{INTRODUCTION}

Less than 1 year after the launch of the bivalent and quadrivalent human papillomavirus
Strengths and limitations of this study

- The study used a previously validated model.

- The analyses used Chinese-specific input data.

- Only analysed individuals without prior infection.

- Used a static model instead of a dynamic model and did not consider herd immunity.

- Did not take into account the prevention of genital warts.

(HPV) vaccines in China, the 9-valent HPV vaccine was also approved by the China Food and Drug Administration in April 2018. Unlike the review processes of bivalent and quadrivalent HPV vaccines that took about 10 years in China, the review process of 9-valent HPV vaccine took a record short period of 9 days. ${ }^{1}$ However, the 9 -valent HPV vaccine was only approved for use among 16-26-year-old girls, whereas the bivalent and quadrivalent HPV vaccines were approved for use among 9-26-year-old boys and girls. ${ }^{2}$

Among the oncogenic HPV types, the bivalent and the quadrivalent vaccines are efficacious against types 16/18, whereas the 9 -valent vaccine provides additional protection against types 31/33/45/52/58. ${ }^{3}$ Both the quadrivalent and the 9 -valent vaccines are also protective against HPV types 6/11, which can cause genital warts. ${ }^{3}$ The costeffectiveness of bivalent and quadrivalent HPV vaccines for the prevention of cervical cancer has been previously analysed in the setting of China. The results of such analyses were favourable to the cost-effectiveness of the bivalent and quadrivalent vaccines compared with no vaccination for the prevention of cervical cancer. ${ }^{4} 5$ However, the previous analyses in the literature were conducted before the introduction of the first HPV vaccine in China. Hence, the prices of HPV 
vaccines in the previous analyses was around $\$ 50$, which did not reflect the present reality. In the meantime, the cost-effectiveness of the 9-valent HPV vaccine in China is still unknown. In light of this, it is important to obtain evidence on the value of the 9-valent vaccine to determine whether it should be used more broadly. Although previous studies have quantified the cost-effectiveness of the 9-valent vaccines compared with alternative vaccines in other countries, ${ }^{3}{ }^{6-9}$ evidence in other healthcare systems is not portable to China for numerous reasons such as different prices, cancer treatment costs and epidemiological profiles. As such, the objective of the current study was to analyse the cost-effectiveness of the 9-valent HPV vaccine for the prevention of cervical cancer among Chinese women from the perspective of private sector purchasers because HPV vaccines are neither publicly funded nor reimbursed by any payers to our knowledge. More specifically, this study pertains to the clinical decision setting of whether it is cost-effective for a Chinese woman without previous infection to use the 9-valent HPV vaccine. We compared the 9-valent vaccine with the quadrivalent vaccines, the bivalent vaccines and no vaccination, respectively. Among these, the comparison with the quadrivalent vaccine forms a specific incremental efficacy evaluation of cervical cancer prevention. Thus, it serves as the primary basis for discussion and conclusion. The comparison with the bivalent vaccine may be complicated by the additional efficacy of preventing nononcogenic HPV types provided by the 9-valent vaccine, whereas the comparison with no vaccination is arguably not incremental. However, the alternative comparisons were necessary to render a comprehensive understanding of the health economic profile of the 9-valent vaccine, which is important because even the bivalent and the quadrivalent vaccines are subject to cost-effectiveness concerns for women living in rural areas of China. ${ }^{10}$

\section{METHODS}

We adapted the Papillomavirus Rapid Interface for Modelling and Economics (PRIME) model in the current analysis. The PRIME model is a health economic model developed by WHO that allows country-specific evaluation of HPV vaccination among women without prior infection of HPV. ${ }^{11}{ }^{12}$ Country-specific cervical cancer incidence, mortality, HPV type distribution and economic data were built in the model. The model developers assessed the quality of country-specific data as either 'satisfactory' or 'unsatisfactory' based on availability of data for each country and quality of methods used in data collection, and the Chinese data were deemed 'satisfactory'. ${ }^{11}$ The model calculates the incremental costs per disabilityadjusted life year (DALY) prevented for vaccinated individuals over a lifetime as well as population outcomes such as cervical cancer prevented and deaths prevented. In addition, the model was validated against previously published HPV vaccine cost-effectiveness studies in the literature. More details of the model have been described elsewhere and in online supplementary appendix $1 .{ }^{11}$

The current analysis only examined incremental costs per DALY prevented for vaccinated individuals. Because the intervention of interest in the current analysis is 9-valent HPV vaccination, we modified the model to use the proportion of cervical cancer that was attributable to HPV types $16 / 18 / 31 / 33 / 45 / 52 / 58$ in China reported by International Agency for Research on Cancer (IARC) HPV Information Centre instead of only the proportion that was attributable to types $16 / 18$ in the original model. ${ }^{12}{ }^{13}$ According to IARC estimates, 92\% of cervical cancer in China were attributable to types $16 / 18 / 31 / 33 / 45 / 52 / 58 .^{13}$

The model also permitted customisation of target age group, efficacy of vaccine (percentage of cervical cancer reduction), vaccine price, vaccine delivery costs, cancer treatment cost, discount rate and disutility values of three cancer-related health states (cancer diagnosis, nonterminal cancer sequelae and terminal cancer). Data in the customised input fields can over-ride the default data. In the current analysis, default data of efficacy of vaccine, discount rate and disutility values were used. It should be noted that several other inputs could be customised in the model including coverage rate (or uptake rate), birth cohort size and cohort size at the vaccination age. Except for coverage rate, these inputs only affect population outcomes that are not of interest in the current analysis and does not affect the results of the cost-effectiveness results for vaccinated individuals. We assumed a coverage rate of $100 \%$ in our analysis such that the mean population result is equivalent to that of an average vaccinated individual.

In the base case analysis, the target age group was 16-year-old girls because this was the youngest group among the current age of licensure of the 9-valent vaccine in China (additional explanation in online supplementary appendix 2). It is noteworthy that this was older than WHO-recommended primary target age window of 9-14 years. ${ }^{14}$ Regardless of vaccination age, the time horizon was set so such that the cohort were followed up to 100 years old. The price of 9-valent HPV vaccine in government procurement catalogue as of December 2018 was used. ${ }^{1516}$ We also assessed the prices at which the incremental costs per DALY prevented were at the cost-effective threshold of three times the 2017 China gross domestic product (GDP) per capita and at the highly cost-effective threshold of once the 2017 China GDP per capita to inform decision makers of the value-based prices. Therefore, the cost-effective threshold and the highly costeffective thresholds are US\$25 920/DALY prevented and US $\$ 8640 /$ DALY prevented, respectively. ${ }^{17}$ Default data of vaccine administration costs per person in China in the PRIME model were used but adjusted to 2017 equivalent using the healthcare component Consumer Price Indices in China. ${ }^{18}$ In addition, cancer treatment costs in 2015 were updated to 2017 US dollars. ${ }^{18} 19$ Input data are listed in table 1. 
Table 1 Input data and model results of the base case analysis of the 9-valent HPV vaccine and the exploratory analyses of the bivalent and quadrivalent HPV vaccines

\begin{tabular}{|c|c|c|}
\hline \multicolumn{3}{|l|}{ Input data } \\
\hline Parameter & Value & Reference/source \\
\hline Vaccination age & 16 years & NA \\
\hline $\begin{array}{l}\text { Percentage of cervical } \\
\text { cancer in China } \\
\text { attributable to types } \\
16 / 18 / 31 / 33 / 45 / 52 / 58\end{array}$ & $92.0 \%$ & 13 \\
\hline $\begin{array}{l}\text { Percentage of cervical } \\
\text { cancer in China } \\
\text { attributable to types } \\
16 / 18\end{array}$ & $69.1 \%$ & 13 \\
\hline $\begin{array}{l}\text { 9-valent vaccine price for } \\
\text { full doses (2017 US\$) }\end{array}$ & $\$ 610$ & 1516 \\
\hline $\begin{array}{l}\text { Quadrivalent vaccine } \\
\text { price for full doses ( } 2017 \\
\text { US\$) }\end{array}$ & $\$ 375$ & 1516 \\
\hline $\begin{array}{l}\text { Bivalent vaccine price for } \\
\text { full doses (2017 US\$) }\end{array}$ & $\$ 273$ & 1516 \\
\hline $\begin{array}{l}\text { Vaccine administration } \\
\text { costs (2017 US\$) }\end{array}$ & $\$ 18$ & $\begin{array}{l}\text { Model default with } \\
\text { inflation adjustment }^{16} \\
18\end{array}$ \\
\hline $\begin{array}{l}\text { Cancer treatment costs } \\
\text { (2017 US\$) }\end{array}$ & $\$ 7183$ & 1819 \\
\hline Efficacy of vaccine & $100 \%$ & Model default \\
\hline Discount rate & $3 \%$ & $\begin{array}{l}\text { Base case } \\
\text { assumption }\end{array}$ \\
\hline $\begin{array}{l}\text { Disutility weight of } \\
\text { cancer diagnosis }\end{array}$ & 0.08 & Model default \\
\hline $\begin{array}{l}\text { Disutility weight of non- } \\
\text { terminal cancer sequelae }\end{array}$ & 0.11 & Model default \\
\hline $\begin{array}{l}\text { Disutility weight of } \\
\text { terminal cancer }\end{array}$ & 0.78 & Model default \\
\hline
\end{tabular}

DALY, disability-adjusted life year;HPV, human papillomavirus; ICER, incremental cost-effectiveness ratio measured as incremental costs per DALY prevented; NA, not applicable.

In one-way sensitivity analyses, age at vaccination (each age between 13 and 26 years), efficacy of vaccine (reduced to $90 \%$ ), cervical cancer incidence of all age groups, cervical cancer mortality, all-cause mortality, cancer treatment costs, disutility of terminal cancer and discount rate ( $1 \%$ and $5 \%)$ were varied to examine the robustness of incremental costs per DALY prevented results. Parameters of interest other than age at vaccination, efficacy and discount rate were increased and decreased by $25 \%$. In the pivotal clinical trial based on which the quadrivalent HPV vaccine was approved by the Chinese regulatory body, the efficacy against cervical intraepithelial neoplasia (CIN) grades $1+$ and $2+$ related to HPV 6/11/16/18 was $100 \%$ at the end of the 12th month. ${ }^{20}$ Also, the efficacy against cervical persistent infection was above $90 \% .{ }^{20}$ Therefore, the efficacy was set to $90 \%$ in the sensitivity analyses to test the impact of possible lower efficacy after vaccination.

\section{Patient and Public Involvement \\ Patients were not involved.}

\section{RESULTS}

The base case results of comparing the 9-valent vaccine with the quadrivalent and bivalent vaccines are shown in table 2 (more detailed information on the basecase results is in online supplementary appendix 3). The incremental costs per DALY prevented compared with the quadrivalent and the bivalent vaccines were US $\$ 35000$ and US $\$ 50455$, respectively. Therefore, the 9 -valent vaccine was not cost-effective when compared with either the quadrivalent or the bivalent vaccine. To be cost-effective, the 9-valent vaccine should be priced at $\$ 550$ and $\$ 450$ for the full doses, respectively. To be highly cost-effective, the price thresholds were $\$ 435$ and $\$ 335$. Furthermore, the 9-valent vaccine should be priced at $\$ 505$ to reach the same ICER as the quadrivalent vaccine when both vaccines were compared with no vaccination. The corresponding price was $\$ 370$ when the comparator was the bivalent vaccine.

The results of comparing with no vaccination are displayed in table 2. The incremental costs per DALY prevented were US $\$ 23012$. This was slightly less than the cost-effective threshold but substantially above the highly cost-effective threshold. In addition, the prices for the 9-valent HPV vaccine to be cost-effective and highly costeffective were $\$ 680$ and $\$ 220$, respectively.

The results of one-way sensitivity analyses are presented in online supplementary appendix 4 . The results of comparing with the quadrivalent and the bivalent vaccines were relatively sensitive to using alternative discount rates. When the discount rate was $1 \%$, the 9-valent vaccine was cost-effective compared with both the quadrivalent and the bivalent vaccines, but not highly costeffective. However, none of the other changes impacted the inference using either the cost-effective threshold or the highly cost-effective threshold. In the comparison with no vaccination, both using alternative discount rates and changing the mortality rate of cervical cancer had substantial impacts on the results. When the discount rate was $5 \%$, the incremental costs per DALY prevented in the comparison of the 9-valent vaccine and no vaccination were $\$ 43,145$, which was above the cost-effective threshold. Similarly, the corresponding result was \$30 246 when the mortality rate of cervical cancer was reduced by $25 \%$, which was also above the cost-effective threshold. The results remained cost-effective in all other scenarios.

\section{DISCUSSION}

In the present analysis, the 9-valent HPV vaccine was not cost-effective for the prevention of cervical cancer among 16-26-year-old Chinese girls without prior HPV 
Table 2 Base case results

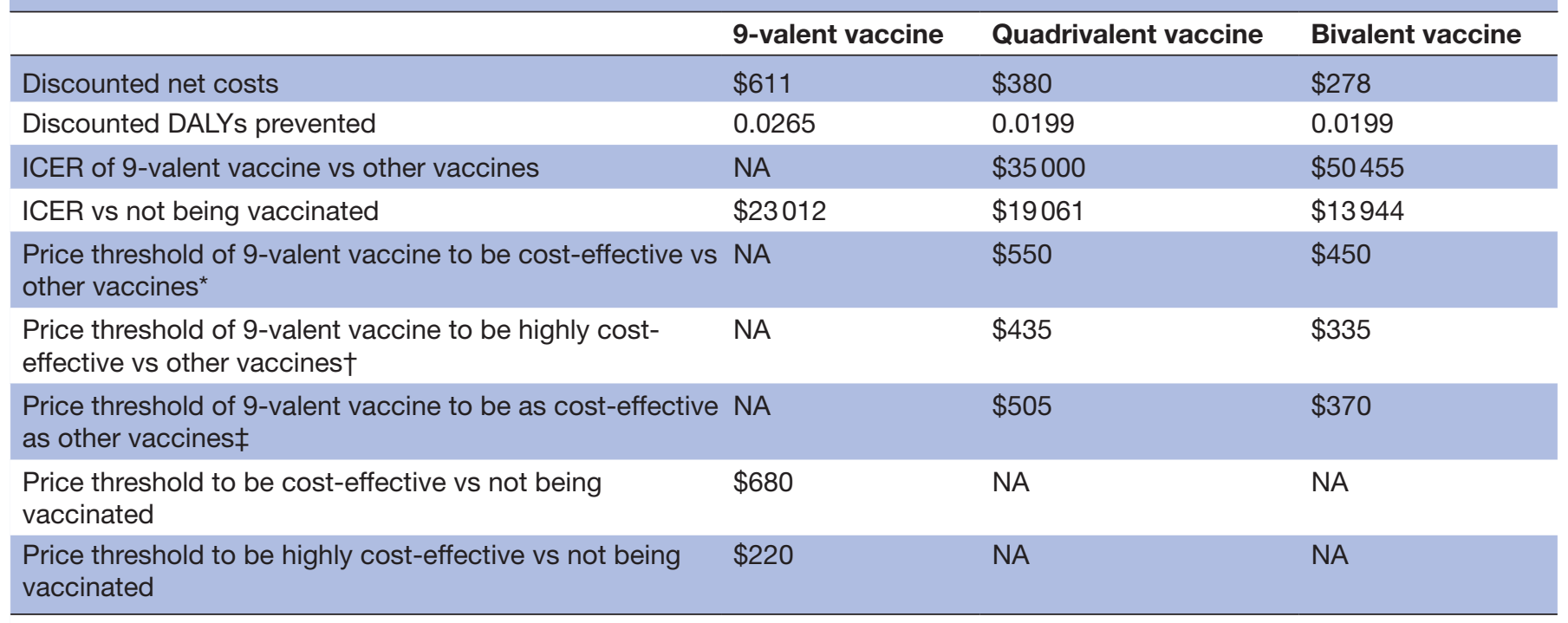

*For example, the 9-valent vaccine should be priced at $\$ 550$ for the full doses to be cost-effective when compared with the quadrivalent vaccine.

†For example, the 9 -valent vaccine should be priced at $\$ 435$ for the full doses to be highly cost-effective when compared with the quadrivalent vaccine.

$\ddagger$ At this price, the 9-valent vaccine is as cost-effective as the quadrivalent vaccine when each of them was compared with not receiving vaccination if the price of the 9 -valent vaccine is $\$ 505$ for the full doses. This is equivalent to saying that the cost-effectiveness of the 9-valent vaccine vs the quadrivalent vaccine is the same as the cost-effectiveness of the quadrivalent vaccine vs not receiving vaccination. The interpretation of the comparison vs the bivalent vaccine is the same.

DALY, disability-adjusted life year; ICER, incremental cost-effectiveness ratio measured as incremental costs per DALY prevented; NA, not applicable.

infection when compared with either the quadrivalent or the bivalent vaccine, which remained so in all the sensitivity analyses except for using a discount rate of $1 \%$. The results suggest that the price of the 9-valent HPV vaccine needs to be adjusted downward to provide more value for Chinese female recipients. Since the highly cost-effective threshold is more stringent, the 9-valent HPV vaccine is also not highly cost-effective in China.

The results are important to the extent that the marginal health gain of investing in the quadrivalent or bivalent vaccine is more than that of the 9-valent vaccine. Although the comparison with the bivalent vaccine may not be fair given that the bivalent vaccine does not protect against warts, the comparison with the quadrivalent vaccine is not subject to the same limitation. As far as cervical cancer is concerned, our results showed that the marginal health gain of an extra dollar in the healthcare budget to be spent on the 9-valent vaccine would be the same as that on the quadrivalent and bivalent vaccine if the 9-valent vaccine were to be priced at $\$ 505$ and $\$ 370$, respectively. In the meantime, a number of Chinese-manufactured bivalent and quadrivalent HPV vaccines are already in the late stage of clinical trials and will likely be marketed at lower prices than the imported counterparts. The entrance of these products will further neutralise the edge of the 9-valent vaccine over the other vaccines in respect to cost-effectiveness. At the current price level, clinicians and policymakers are advised to educate potential vaccine recipients and keep them informed when suggesting vaccination.

The 9-valent HPV vaccine was cost-effective but not highly cost-effective for the prevention of cervical cancer among 16-26-year-old Chinese girls without prior HPV infection when compared with no vaccination. The results were robust to changes in important parameters except for discount rates and cervical cancer mortality.

While it is both intuitive and theoretically founded to compare the 9-valent vaccine only with the quadrivalent and the bivalent vaccines, it should be noted that further comparing the 9-valent vaccine with no vaccination may provide additional insights when the standard of practice is absent. Indeed, only comparing the 9-valent vaccine with the other HPV vaccines suffices to inform decisionmaking if HPV vaccination is already the standard of practice and the decision should pertain to incremental cost-effectiveness in relation to the standard of practice. While this might be true in high-income countries, it is not necessarily the case in China. Most Chinese women, regardless of age, have not been inoculated with any HPV vaccine. Specifically, the total number of HPV vaccine doses released by the National Institutes for Food and Drug Control in all batches as of September 2018 was merely 6 million, ${ }^{21}$ indicating the maximum number of women in China that would have received at least one dose of HPV vaccine. Hence, the scenario that a portion of the individuals would only consider either receiving 
the 9-valent vaccine or not being vaccinated should not be ruled out. For these individuals, the comparison of the 9-valent vaccine with no vaccination is relevant for decision making. As such, we pertain to the comparison with the quadrivalent vaccine as the primary analysis but also provide exploratory results of comparing with no vaccination.

It is important to note that the results of age-related sensitivity analyses do not necessarily suggest that vaccination is more cost-effective at older ages. Smaller ICERs at older ages are mainly caused by fewer years of discounting the benefits (see online supplementary appendix 5 ).

The cost-effectiveness profile of the 9-valent vaccine based on the present analysis contrasts that in several developed countries. A study found that the 9-valent vaccine was cost-effective compared with the quadrivalent vaccine among 12-26-year-old girls in the USA if the additional acquisition costs per dose was no more than US $\$ 13 .{ }^{3}$ Their finding was confirmed by another US study. ${ }^{6}$ An Australian study also showed that the 9-valent vaccine was a cost-effective alternative to the quadrivalent vaccine for 12-year-old girls if the additional costs per dose was under AUS $\$ 36 .{ }^{7}$ In Canada, Italy and Spain, the corresponding numbers were CAN $\$ 24, € 16$ and $€ 16 .{ }^{8922}$ These numbers were generally consistent with the realworld price differences in the public sectors of the aforementioned countries. ${ }^{389}$ However, the gaps between the prices of the 9-valent and quadrivalent vaccines in these markets were substantially smaller than that in China, which is likely the main reason of the inconsistent costeffectiveness profiles. This highlights the importance of adjusting the price of the 9-valent vaccine in China from the value perspective.

The current analysis is subject to several limitations. First, our analysis did not model catch-up immunisation for those individuals who have already had prior infections. ${ }^{12}$ It is reasonable to expect that the incremental benefit of the 9-valent vaccine is smaller among these individuals. Second, the analysis only considered the health benefits of preventing cervical cancer but not the benefits of preventing genital warts. Taking into account the prevention of genital warts will favour the 9 -valent vaccine over the bivalent vaccine and no vaccination. In addition to these limitations, the model was also subject to other limitations that do not necessarily undermine the validity of the current results. For example, the model does not evaluate vaccination combined with cervical screening programme or assess herd immunity due to vaccination. ${ }^{12}$ To the extent that the concentration of the present study is shedding light on the cost-effectiveness profiles of vaccines, including screening may obscure the focus as well as create confusion in the selection of decision perspective. Also, the model is only appropriate to evaluate vaccination among 9-13-year-old girls if the population outcomes are of interest because some individuals in a cohort of older ages may have been previously infected. In theory, these limitations do not affect the economic evaluation of vaccinating an individual who is known to have no previous infection and decides to accept immunisation. Future economic evaluations should examine screening and vaccination strategies in which a portion of the target population was infected. More, Chinesemanufactured vaccines in future may affect the pricing of marketed products and the CEA should be updated accordingly. Even more, the efficacy of the vaccine was assumed to stay fully protective throughout the time horizon. This does not necessarily reflect the reality. However, the impact of this limitation is unclear since it affects both the 9-valent vaccine and the other vaccines.

\section{CONCLUSIONS}

In conclusion, the 9-valent HPV vaccine is not cost-effective when compared with the quadrivalent vaccine for young girls in China who had not been previously infected with HPV at its current price. It is also not cost-effective when compared with the bivalent vaccine, although it is marginally cost-effective yet not highly cost-effective when compared with no vaccination. Given these results, policymakers and clinicians should be conservative to expand the use of the 9-valent HPV vaccines in China unless the price is reduced. In addition, it is important that the clinicians discuss the economic profile of the 9-valent HPV vaccine to keep health-seeking individuals informed. More, public health professionals should be cautious about using the 9-valent vaccine as the primary choice at its current price if HPV vaccines are to be provided as public goods nationally.

Contributors Design and data collection: YJ, WN and JW. Analysis and interpretation: YJ, WN and JW. Drafting the manuscript: YJ. Reviewing and revising the manuscript: WN and JW.

Funding The authors have not declared a specific grant for this research from any funding agency in the public, commercial or not-for-profit sectors.

Competing interests None declared.

Patient consent for publication Not required.

Provenance and peer review Not commissioned; externally peer reviewed.

Data availability statement Data are available in a public, open access repository. All data relevant to the study are included in the article or uploaded as supplementary information.

Open access This is an open access article distributed in accordance with the Creative Commons Attribution Non Commercial (CC BY-NC 4.0) license, which permits others to distribute, remix, adapt, build upon this work non-commercially, and license their derivative works on different terms, provided the original work is properly cited, appropriate credit is given, any changes made indicated, and the use is non-commercial. See: http://creativecommons.org/licenses/by-nc/4.0/.

ORCID iD

Jing Wu http://orcid.org/0000-0002-6012-2785

\section{REFERENCES}

1 Liu A. 9 days for Gardasil 9: China hands out landmark NOD with lightning speed, 2018. Available: https://www.fiercepharma.com/ pharma-asia/9-days-for-gardasil-9-china-hands-out-landmarkconditional-nod-lightning-speed

2 HPV vaccine becomes available in China for women between 16 to 26 years old, 2018. Available: https://www.firstwordpharma.com/ node/1560878?tsid=6 
3 Chesson HW, Markowitz LE, Hariri S, et al. The impact and costeffectiveness of nonavalent HPV vaccination in the United States: estimates from a simplified transmission model. Hum Vaccin Immunother 2016:12:1363-72.

4 Levin CE, Sharma M, Olson Z, et al. An extended cost-effectiveness analysis of publicly financed HPV vaccination to prevent cervical cancer in China. Vaccine 2015;33:2830-41.

5 Canfell K, Shi J-F, Lew J-B, et al. Prevention of cervical cancer in rural China: evaluation of HPV vaccination and primary HPV screening strategies. Vaccine 2011;29:2487-94.

6 Durham DP, Ndeffo-Mbah ML, Skrip LA, et al. National- and statelevel impact and cost-effectiveness of nonavalent HPV vaccination in the United States. Proc Natl Acad Sci U S A 2016;113:5107-12.

7 Simms KT, Laprise J-F, Smith MA, et al. Cost-effectiveness of the next generation nonavalent human papillomavirus vaccine in the context of primary human papillomavirus screening in Australia: a comparative modelling analysis. Lancet Public Health 2016;1:e66-75

8 De La Fuente J, Hernandez Aguado JJ, San Martín M, et al. Estimating the epidemiological impact and cost-effectiveness profile of a nonavalent HPV vaccine in Spain. Hum Vaccin Immunother 2019;15:1949-61.

9 Mennini FS, Bonanni P, Bianic F, et al. Cost-effectiveness analysis of the nine-valent HPV vaccine in Italy. Cost Eff Resour Alloc 2017;15:11.

10 Yin Y. HPV vaccination in China needs to be more cost-effective. Lancet 2017;390:1735-6.

11 Jit M, Brisson M, Portnoy A, et al. Cost-effectiveness of female human papillomavirus vaccination in 179 countries: a PRIME modelling study. Lancet Glob Health 2014;2:e406-14.

12 Hickman M, Jit M, Hutubessy R. Papillomavirus rapid interface for modelling and economics tool user manual, 2014. Available: http://
primetool.org/wp-content/uploads/documents/PRIME_Tool_Manual_ v2.pdf

13 World Health Organization. China: human papillomavirus and related cancers, fact sheet 2017, 2017. Available: http://www.hpvcentre.net/ statistics/reports/CHN_FS.pdf

14 World Health Organization. Electronic address: sageexecsec@who. int. Human papillomavirus vaccines: WHO position paper, May 2017-Recommendations. Vaccine 2017;35:5753-5.

15 Information of drug winning Bid, 2018. Available: https://data.yaozh. com/yaopinzhongbiao

16 XE Currency Table: USD - US Dollar, 2017. Available: https://www.xe. com/currencytables/

17 International Monetary Fund. GDP per capita, current prices - U.S. dollars per capita, 2018. Available: http://www.imf.org/external/ datamapper/NGDPDPC@WEO/OEMDC/ADVEC/WEOWORLD

18 National Bureau of Statistics of China. Consumer price indices, healthcare, 2017. Available: http://data.stats.gov.cn/english/adv.htm? $m=$ advquery\&cn $=A 01$

19 Mo X, Gai Tobe R, Wang L, et al. Cost-effectiveness analysis of different types of human papillomavirus vaccination combined with a cervical cancer screening program in mainland China. BMC Infect Dis 2017;17:502.

20 Wei L, Xie X, Liu J, et al. Efficacy of quadrivalent human papillomavirus vaccine against persistent infection and genital disease in Chinese women: a randomized, placebo-controlled trial with 78-month follow-up. Vaccine 2019;37:3617-24.

21 National Institutes for Food and Drug Control. Batch-release inquiry of biological products, 2018. Available: http://www.nifdc.org.cn/ CL0694/

22 Drolet M, Laprise J-F, Boily M-C, et al. Potential cost-effectiveness of the nonavalent human papillomavirus (HPV) vaccine. Int J Cancer 2014;134:2264-8. 